\title{
Analytical Formulation of a Quasi-periodic Undulator
}

\author{
M. Takao, S. Hashimoto, S. Sasaki and Y. Miyahara, \\ SPring-8, Kamigori, Ako-gun, Hyogo 678-12, Japan
}

\section{Abstract}

Recently two of the present authors (S. H. and S. S.) introduced the concept of a new class of undulators, a quasi-periodic array of magnet poles, to discriminate the rational higher harmonics of radiation that are harmful in some synchrotron experiments. In this paper the analytical formula of the radiation spectrum from the quasi-periodic undulator is reported.

\section{Introduction}

Ordinary undulators consist of a periodic array of magnet poles of alternating polarity. The radiation emitted in each magnet pole interferes with each other, producing enhanced emission at a fundamental frequency and its harmonics. Since a mixture of the harmonics degrades the ratio of signal to noise in many experiments, the higher harmonics are required to be eliminated. In the high energy region of $\mathrm{x}$-rays above $30 \mathrm{keV}$, however, it is practically difficult to exclusively pick up the fundamental radiation. Hashimoto and Sasaki proposed a new undulator, which comprises a quasi-periodic array of magnet poles [1], [2] and will be called "Quasi-periodic undulator" (hereafter, referred to as QPU). No rational higher harmonics of the fundamental frequency are contained in the radiation from the QPU. Here we analytically formulate the QPU radiation spectrum.

In a normal planer periodic undulator (PU) an electron moves sinusoidally in the horizontal plane. It takes the time $\lambda_{U} /\left(c \bar{\beta}_{z}\right)$ that the electron traverses one period of the undulator $\lambda_{U}$, where $\bar{\beta}_{z}$ is the average longitudinal velocity. During this time the light travels the distance $L=\lambda_{U} / \bar{\beta}_{z}$ along the undulator axis, so that the light emitted by an electron at a top of the sinusoidal motion precedes the one emitted at the next top by the phase $\Delta \phi=2 \pi \omega / \omega_{1}$. Here $\omega$ is the angular frequency of the light, and $\omega_{1}$ is the resonant frequency of the undulator radiation given by $\omega_{1}=2 \gamma^{2} \omega_{U} /\left(1+K^{2} / 2\right)$ with the undulator angular frequency $\omega_{U}=2 \pi c / \lambda_{U}$. Gathering the radiations from the individual periods, one finds that the intensity is proportional to [3], [4], [5]

$$
\sum_{m=0}^{N-1} \exp \left(2 \pi i m \frac{\omega}{\omega_{1}}\right)=e^{\pi i(N-1) \frac{\omega}{\omega_{1}}} \frac{\sin \left(N \pi \frac{\omega}{\omega_{1}}\right)}{\sin \left(\pi \frac{\omega}{\omega_{1}}\right)},
$$

where $N$ is the number of periods of the undulator. This implies that the intensity of the undulator radiation is strengthened at $\omega=n \omega_{1}$ with an integer $n$ and that the spectrum has the harmonic structure.

The explicit formula of the radiation spectrum from the PU is well-known and expressed by infinite series of Bessel functions [6], [7], [8]. In the next section we briefly review the irrational harmonic structure of the radiation spectrum from a QPU and give the explicit expression.

\section{Radiation Spectrum for a QPU}

The $m$ th quasi-periodic lattice point is represented as [1], [2], [9]

$$
\hat{z}_{m}=m-(\tan \alpha-1)+(\tan \alpha-1)\left[\frac{\tan \alpha}{1+\tan \alpha} m+1\right],
$$

where $\tan \alpha$ is the tangent of the inclination angle of a 1D quasilattice against a 2D square lattice. The symbol $[\cdots]$ represents the greatest integer operator. The first term on the right hand side in Eq. (2) corresponds to a periodic component of spacing between the lattice points, the second term represents the constant translation of lattice points, which moves the initial lattice point $\hat{z}_{0}$ to the origin, and as $m$ is increased the third term quasiperiodically increases by $(\tan \alpha-1)$ due to the irrational nature of $\tan \alpha$. Hence the distance between any two consecutive positions $\left(\hat{z}_{m}-\hat{z}_{m-1}\right)$ takes a value of 1 or $\tan \alpha$, forming a quasi-periodic array.

A basic magnetic structure for the planer QPU can be realized by aligning positive and negative magnet poles alternately at the 1D quasi-lattice points designated by Eq. (2) [1], [2]. From the symmetry of the 2D square lattice, where a 1D quasi-lattice is embedded, we can restrict $0<\tan \alpha<1$ without loss of generality. Thus we denotes the two distances between the quasilattice points as $d, d^{\prime}(=d / \tan \alpha>d)$. To realize a QPU, the length of the magnet block $w$ should be shorter than the distance $d$.

As in a regular PU we here assume that the magnetic field $B_{y}(z)$ of the transverse QPU with $N^{\prime}$ poles has the sinusoidal dependence

$$
B_{y}(z)=\sum_{m=0}^{N^{\prime}-1} B_{0}(-1)^{m} \operatorname{Cos}\left[\frac{\pi}{w}\left(z-z_{m}\right)\right],
$$

where $B_{0}$ is the peak magnetic field and $z_{m}\left(=d^{\prime} \hat{z}_{m}\right)$ the center of the $m$ th magnet region. The function $\operatorname{Cos}(\alpha)$ is here defined to take $\cos (\alpha)$ for $-\pi / 2 \leq \alpha<\pi / 2$ and 0 otherwise. The magnetic field distribution with $w=d$ is shown in Fig. 1. For the sake of later convenience we here define analogue of the wave number, $k_{0}=\pi / w$, and the undulation parameter, $K=\left(e B_{0}\right) /\left(m_{0} c k_{0}\right)$, in the QPU.

In estimating the phase interference in the radiation from the QPU, we must sum up the phase differences of the individual magnet poles instead of the periods in the PU. Then the function that contains the phase interference of the QPU with $N^{\prime}$ magnet poles is

$$
Q_{\ell}(\omega)=\frac{1}{N^{\prime}} \sum_{m=0}^{N^{\prime}-1} \exp \left[i \pi\left\{\frac{\omega}{\omega_{2}}\left(\hat{z}_{m}-\eta m\right)-\ell m\right\}\right],
$$

where

$$
\omega_{2}=\frac{w}{d^{\prime}} \frac{2 \gamma^{2} \omega_{0}}{1+K^{2}+\left(\gamma \theta_{0}\right)^{2}}
$$




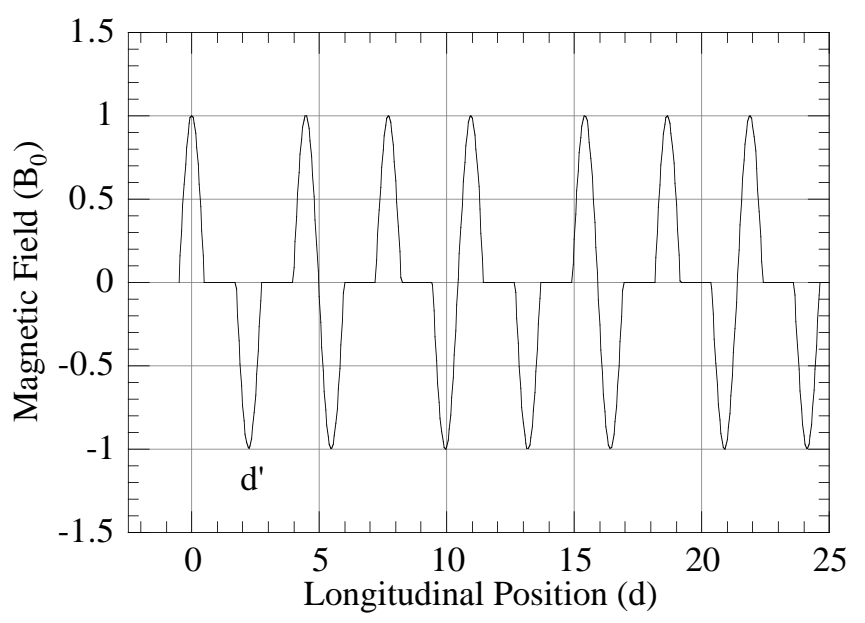

Figure. 1. Magnetic field distribution along the axis of the quasiperiodic undulator.

$$
\eta=\frac{w}{2 d^{\prime}} \frac{K^{2}}{1+K^{2}+\left(\gamma \theta_{0}\right)^{2}} .
$$

In the exponent of Eq. (4) the term $i \pi\left(\omega / \omega_{2}\right) \hat{z}_{m}$ is the counterpart of the phase difference in the PU $2 i \pi m\left(\omega / \omega_{2}\right)$. The factor 2 in the phase of the PU reflects the fact that one period of a PU consists of two magnet poles. Since in the free spaces between the magnet regions the electron possesses the transverse velocity, the phase of the electron against the light delays while the electron runs through the free spaces. The second term in the exponent of Eq. (4) containing $\eta$ corresponds to this phase delay. The index $\ell$ of $Q_{\ell}$ represents the order of the Bessel expansion of the sinusoidal phase motion in the magnet regions.

Since for even $\ell$ the second term in the exponent in Eq. (4) is an integer multiple of $2 \pi i$ and then vanishes in the exponentiation, the summation $Q_{\ell}(\omega)$ can be simplified as

$$
Q_{\ell=\mathrm{even}}(\omega)=\frac{1}{N^{\prime}} \sum_{m=0}^{N^{\prime}-1} e^{i \pi \frac{\omega}{\omega_{2}}\left(\hat{z}_{m}-\eta m\right)}
$$

which represents the Fourier transform of the quasi-periodic lattice except for the additional term $\eta m$. Following the description of the quasi-crystal [9], we can derive the approximated expression of $Q_{\ell}(\omega)$. For large $N^{\prime}$ approximation, Eq. (7) can be easily rewritten as [9]

$$
Q_{\ell=\mathrm{even}}(\omega)=\sum_{p, q} e^{i X_{p q} / 2} \frac{\sin \left(X_{p q} / 2\right)}{X_{p q} / 2} \delta\left(\pi \frac{\omega}{\omega_{2}}-k_{p q}\right),
$$

where

$$
\begin{aligned}
k_{p q} & =2 \pi\left(p+q \frac{\tan \alpha}{1+\tan \alpha}\right) /\left(\frac{1+\tan ^{2} \alpha}{1+\tan \alpha}-\eta\right), \\
X_{p q} & =2 \pi q-(\tan \alpha-1) k_{p q} .
\end{aligned}
$$

This implies that bright peaks occur at the discrete values of $k_{p q}$ 's where $X_{p q}$ 's are small and that intense peak positions $(p, q)$ should be associated with the Fibonacci sequence [9]. Hence, in general, there appears no rational higher harmonic in the radiation spectrum from the QPU.
In the case of odd $\ell$, the summation $Q_{\ell}(\omega)$ is reduced to

$$
Q_{\ell=\mathrm{odd}}(\omega)=\frac{1}{N^{\prime}} \sum_{m=0}^{N^{\prime}-1} e^{i \pi \frac{\omega}{\omega_{2}}\left(\hat{z}_{m}-\eta m\right)-i \pi m}
$$

which implies that it corresponds to the Fourier transform of the quasi-lattice with positive and negative matters, since the additional phase factor $\exp (-i \pi m)$ alternately changes the sign as $m$ increasing. Then the peak position $k_{p q}$ is shifted by the second term of Eq. (11) from the positions of $\ell=$ even, and given by

$$
k_{p q}=\left[2 \pi\left(p+q \frac{\tan \alpha}{1+\tan \alpha}\right)-\pi\right] /\left(\frac{1+\tan ^{2} \alpha}{1+\tan \alpha}-\eta\right) .
$$

In both the cases the resonant frequency of the synchrotron radiation from the QPU $\omega_{p q}$ is represented as

$$
\omega_{p q}=\frac{k_{p q}}{\pi} \omega_{2}
$$

It is emphasized that the resonant frequency of the radiation from QPU $\omega_{p q}$ has the extra $K$ dependence through $\eta$ in $k_{p q}$ in addition to through $\omega_{2}$ while the one from a PU is a simple integer multiple of $\omega_{1}$.

Evaluating the radiation intensity from one magnet pole, we can derive the explicit form of the spectrum formula

$$
\begin{aligned}
\frac{d^{2} I(\omega)}{d \omega d \Omega} & =\frac{e^{2} N^{\prime 2} \gamma^{2}}{16 \pi \epsilon_{0} c} \sum_{p, q}\left(\left|\gamma \theta_{0} \cos \phi_{0} F_{p q}-\frac{K}{2} G_{p q}\right|^{2}\right. \\
& \left.+\left|\gamma \theta_{0} \sin \phi_{0} F_{p q}\right|^{2}\right) H_{p q}(\omega)
\end{aligned}
$$

where, for an odd mode

$$
\begin{aligned}
& F_{p q}=\left[\frac{2 \tilde{k}_{p q}}{1+K^{2} / 2+\left(\gamma \theta_{0}\right)^{2}}\right. \\
& \left.\times \sum_{\ell=\text { odd }} \frac{S_{\ell}^{(0)}\left(\tilde{k}_{p q}\right)}{\frac{\pi}{2}\left(\tilde{k}_{p q}-\ell\right)}-\frac{4}{\pi} C\right] \cos \left(\frac{\pi}{2} \tilde{k}_{p q}\right), \\
& G_{p q}=\left[\frac{2 \tilde{k}_{p q}}{1+K^{2} / 2+\left(\gamma \theta_{0}\right)^{2}}\right. \\
& \left.\times \sum_{\ell=\text { odd }} \frac{S_{\ell}^{(1)}\left(\tilde{k}_{p q}\right)+S_{\ell}^{(-1)}\left(\tilde{k}_{p q}\right)}{\frac{\pi}{2}\left(\tilde{k}_{p q}-\ell\right)}-\frac{8}{\pi} B\right] \cos \left(\frac{\pi}{2} \tilde{k}_{p q}\right),
\end{aligned}
$$

and for an even mode

$$
\begin{aligned}
& F_{p q}= {\left[\frac{2 \tilde{k}_{p q}}{1+K^{2} / 2+\left(\gamma \theta_{0}\right)^{2}}\right.} \\
&\left.\times \sum_{\ell=\text { even } \frac{S_{\ell}^{(0)}\left(\tilde{k}_{p q}\right)}{2}\left(\tilde{k}_{p q}-\ell\right)}-\frac{4}{\pi} B\right] \sin \left(\frac{\pi}{2} \tilde{k}_{p q}\right), \\
& G_{p q}= {\left[\frac{2 \tilde{k}_{p q}}{1+K^{2} / 2+\left(\gamma \theta_{0}\right)^{2}}\right.} \\
&\left.\times \sum_{\ell=\text { even }} \frac{S_{\ell}^{(1)}\left(\tilde{k}_{p q}\right)+S_{\ell}^{(-1)}\left(\tilde{k}_{p q}\right)}{\frac{\pi}{2}\left(\tilde{k}_{p q}-\ell\right)}-\frac{8}{\pi} C\right] \sin \left(\frac{\pi}{2} \tilde{k}_{p q}\right) .
\end{aligned}
$$


In the spectral formula (14) $H_{p q}\left(\omega / \omega_{1}\right)$ is the structural function given by

$$
H_{p q}(\omega)=\left[\frac{\sin \left(X_{p q} / 2\right)}{X_{p q} / 2}\right]^{2} \delta\left(\omega-\frac{k_{p q}}{\pi} \omega_{2}\right) .
$$

In Eqs. (15)-(18)

$$
\begin{aligned}
\tilde{k}_{p q} & =\frac{d^{\prime}\left[1+K^{2} / 2+\left(\gamma \theta_{0}\right)^{2}\right]}{\pi w\left[1+K^{2}+\left(\gamma \theta_{0}\right)^{2}\right]} k_{p q}, \\
S_{\ell}^{(p)}(x) & =\sum_{n=-\infty}^{\infty} J_{n}\left(\xi_{z} x\right) J_{2 n+\ell+p}\left(\xi_{x} x\right)
\end{aligned}
$$

with

$$
\begin{aligned}
\xi_{x} & =\frac{2 K \gamma \theta_{0} \cos \phi_{0}}{1+K^{2} / 2+\left(\gamma \theta_{0}\right)^{2}}, \\
\xi_{z} & =\frac{K^{2}}{4\left[1+K^{2} / 2+\left(\gamma \theta_{0}\right)^{2}\right]} .
\end{aligned}
$$

In the form factors (15)-(18) the terms $B$ and $C$, given by

$$
\begin{aligned}
B & =\frac{1+K^{2}+\left(\gamma \theta_{0}\right)^{2}}{\left[1+K^{2}+\left(\gamma \theta_{0}\right)^{2}\right]^{2}+\left(2 K \gamma \theta_{0} \cos \phi_{0}\right)^{2}}, \\
C & =\frac{2 K \gamma \theta_{0} \cos \phi_{0}}{\left[1+K^{2}+\left(\gamma \theta_{0}\right)^{2}\right]^{2}+\left(2 K \gamma \theta_{0} \cos \phi_{0}\right)^{2}},
\end{aligned}
$$

are understood as the contributions from the radiation in the free spaces between the magnet poles in the QPU.

\section{Concluding Remarks}

We derived the analytical formula of the radiation from the QPU (14) under the assumption that the magnetic field is given by Eq. (3).

It is worth emphasizing that the intensity of the even modes vanishes on axis as seen in the case of the PU. This is because $G_{p q}$, consisting of $S_{\ell}^{( \pm 1)}$ for even $\ell$ and $C$, vanishes on axis $\left(\theta_{0}=0\right)$. Furthermore, note that, although one infinite Bessel series $S_{\ell}^{(m)}$ for some $\ell$ corresponds to one peak in the radiation spectrum of the PU, a peak intensity of the QPU comes from all the even series or the odd series.

To confirm the validity of the analytical formula, we compare it with the numerical computation of the radiation spectrum from a QPU with the magnetic field given by Eq. (3). Figure. 2 shows the radiation spectra of the QPU with $K=1.0$ given by the numerical and the analytical calculations. Here we take $w=d$ and $\tan \alpha=1 / \sqrt{5}$. In the numerical calculation we assume the number of poles to be 100. The full circles in Figure. 2 represent the bright peaks of the spectrum designated by the generalized Fibonacci integers. Thus one is convinced that the analytical formula for the radiation from the QPU correctly gives the peak position in the spectrum and the peak intensity.

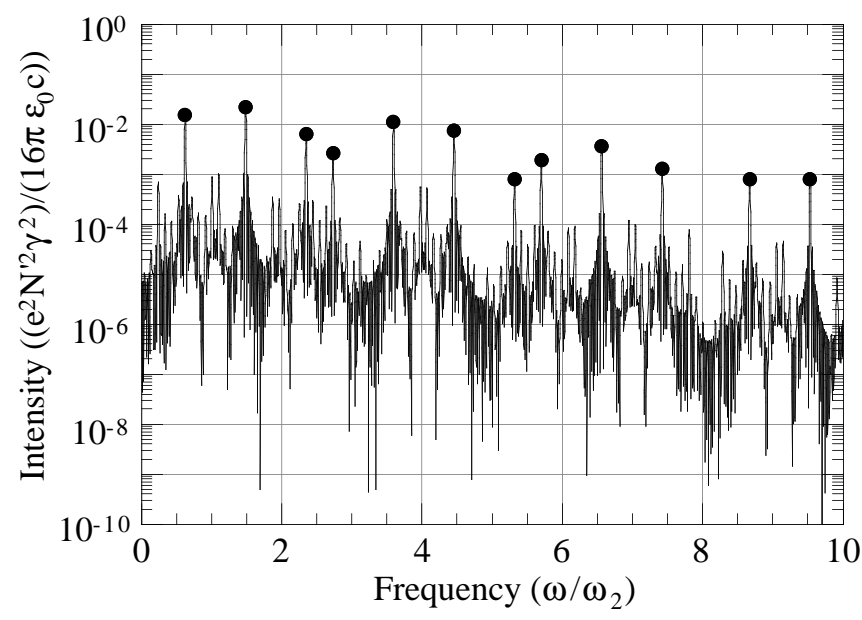

Figure. 2. Radiation spectra from the quasi-periodic undulator with $K=1.0$. The solid curve indicates the numerically computed spectrum and the dots correspond to the peaks given by the analytical formula.

\section{References}

[1] S. Hashimoto and S. Sasaki, JAERI-M Report 94-055 (1994).

[2] S. Sasaki, S. Hashimoto, H. Kobayashi, M. Takao and Y. Miyahara, in Proc. of Inter. Conf. of Synchrotron Radiation Instrumentation '94, New York, U.S.A., 1994.

[3] R. Tatchyn and I. Lindau, Nucl. Instrum. Methods 222 (1984), 14.

[4] R. Tachyn, A.D. Cox and S. Quadri, in Proc. of Inter. Conf. of Insertion Devices for Synchrotron Sources SPIE 582 (1986), 47.

[5] R.P. Walker, Nucl. Instrum. Methods 335 (1993), 328.

[6] H. Kitamura, Jpn. J. Appl. Phys. 19 (1980), L185.

[7] S. Krinsky, Nucl. Instrum. Methods 172 (1980), 73.

[8] S. Krinsky, M.L. Perlman and R.E. Watson, "Characteristics of Synchrotron Radiation and of Its Sources", in "Handbook on Synchrotron Radiation" ed. E.-E. Koch (NorthHolland Publishing Company, Amsterdam, 1983), p.65.

[9] D. Levine and P.J. Steinhardt, Phys. Rev. B34 (1986) 596. 